\title{
Dark brown is the more virulent of the switch phenotypes of Candida glabrata
}

Correspondence

David R. Soll

david-soll@uiowa.edu

Received 14 May 2008

Revised 4 August 2008

Accepted 11 August 2008

\author{
Thyagarajan Srikantha, Karla J. Daniels, Wei Wu, Shawn R. Lockhart, \\ Song Yi, Nidhi Sahni, Ning Ma and David R. Soll
}

Department of Biology, The University of lowa, lowa City, IA 52242, USA

\begin{abstract}
Candida glabrata undergoes reversible, high-frequency core switching between phenotypes that include dark brown (DB), light brown (LB) and white (Wh). These phenotypes in turn can switch to the irregular wrinkle (IWr) phenotype. Natural isolates, however, express predominantly the DB phenotype, leading to the hypothesis that it has a colonization advantage over the other switch phenotypes. Using the mouse model of systemic infection, results are presented which support this hypothesis. DB has an advantage over other switch phenotypes in colonizing the two major target organs in the mouse model, the spleen and liver. A time-course study reveals that colonization of the major target organs occurs very rapidly (within $2 \mathrm{~h}$ ) after host injection, and that the DB advantage for spleen and liver colonization is immediate. The DB advantage is maintained during clearing from spleen, liver and kidneys, and during delayed transient brain colonization. These results demonstrate that DB has a colonization advantage over other switch phenotypes, and that the switch phenotype expressed by a colonizing population therefore plays a fundamental role in virulence. It is therefore essential that switching be considered in both in vivo and in vitro studies of C. glabrata virulence.
\end{abstract}

\section{INTRODUCTION}

In Candida glabrata, there are two high-frequency switching systems, core and irregular wrinkle (Lachke et al., 2000, 2002). In the core system, cells switch among four phenotypes distinguishable by differences in the brown coloration of colonies grown on agar containing $1 \mathrm{mM}$ $\mathrm{CuSO}_{4}$. The core phenotypes are white (Wh), light brown (LB), dark brown (DB) and very dark brown (vDB). Although core switching was first observed and defined on agar containing $\mathrm{CuSO}_{4}$, it has been shown to occur in the absence of $\mathrm{CuSO}_{4}$ (Lachke et al., 2002) and can also be distinguished by graded pink coloration when grown on agar containing phloxine B (Lachke et al., 2002). Cells expressing any of the core phenotypes can also switch to the irregular wrinkle (IWr) phenotype, then back to the original core phenotype (Lachke et al., 2002).

We previously demonstrated in limited plating experiments that 25 of 62 clinical isolates of C. glabrata (40\%) underwent core switching (Lachke et al., 2002). Strains that had not been observed to switch in the initial experiments did so in larger-scale plating experiments. This led us to the conclusion that the great majority of clinical isolates undergo core switching. In natural isolates, the most commonly expressed core phenotype has been found to be

Abbreviations: DB, dark brown; IWr, irregular wrinkle; LB, light brown; vDB, very dark brown; Wh, white.
DB (50\%) and the second most commonly expressed LB (30\%) (Lachke et al., 2002). Wh and vDB of the core system, and IWr of the alternative system, represent minor phenotypes. In an analysis of primary samples from different body locations of three vaginitis patients with $C$. glabrata infections, DB was the dominant phenotype (Brockert et al., 2003). In one patient, however, while DB dominated in the vaginal cavity, Wh dominated in the oral cavity (Brockert et al., 2003). DNA fingerprinting demonstrated that the DB and Wh isolates from this patient were core phenotypes of the same strain. These results indicated that although DB represents the dominant phenotype in nature, in certain situations other switch phenotypes dominate in particular body locations.

The cumulative results, therefore, indicate that the DB phenotype has, overall, a colonization advantage over other phenotypes for host niches. In Candida albicans, the white phenotype of the white-opaque switching system, which represents the most prevalent switch phenotype of clinical isolates that are naturally MTL-homozygous (Lockhart et al., 2005), has been demonstrated to have an advantage over the opaque phenotype in colonizing the kidney, the major target organ, in the mouse model of systemic infection (Kvaal et al., 1997, 1999). We therefore similarly tested the hypothesis that C. glabrata cells expressing the DB phenotype had an advantage by first comparing the levels of colonization of spleen, liver and kidney of DB with 
those of the phenotypes LB, Wh and IWr. We next performed competition experiments by injecting equal numbers of $\mathrm{DB}$ and either Wh or IWr into mice and assessing the levels of colonization of the co-injected phenotypes. Finally, we performed a time-course of Wh or DB colonization of the kidney, liver, spleen and brain. Our results demonstrate that the DB phenotype has a competitive advantage over other switch phenotypes in colonizing the major target organs in the mouse model, which is consistent with its prevalence among clinical isolates.

\section{METHODS}

Strains and culture conditions. C. glabrata strains 35B11 (a), $1480.49(\alpha)$ (Lachke et al., 2002) and derivatives (see below) were stored in $20 \%(\mathrm{v} / \mathrm{v})$ glycerol at $-80{ }^{\circ} \mathrm{C}$. Cells from storage cultures were plated on YPD agar plates $[1.5 \%(\mathrm{w} / \mathrm{v})$ agar, $2 \%(\mathrm{w} / \mathrm{v})$ Bacto Peptone, $2 \%(\mathrm{w} / \mathrm{v})$ glucose, $1 \%(\mathrm{w} / \mathrm{v})$ yeast extract] supplemented with $1 \mathrm{mM} \mathrm{CuSO}_{4}$ and incubated at $25{ }^{\circ} \mathrm{C}$. Four-day-old colonies were then used for experimental purposes. For monitoring phenotypic switching, cells were plated at a density of 40 c.f.u. per plate on YPD agar containing $1 \mathrm{mM} \mathrm{CuSO}_{4}$ (Lachke et al., 2000).

Virulence in a systemic mouse model. Injections were performed as described by Lockhart et al. (2005) for C. albicans, but with minor modifications. Cells from 10 colonies of a four-day culture were pooled and resuspended in Gibco PBS (Invitrogen) at a concentration of $8 \times 10^{7}$ cells $\mathrm{ml}^{-1}$. In the case of IWr, cell preparations were sonicated, resulting in $60 \%$ singlets, $20 \%$ doublets and $20 \%$ triplets. An inoculum of $2 \times 10^{7}$ cells (or cell compartments) in $0.25 \mathrm{ml} \mathrm{PBS}$ was injected into the tail vein of each of a series of six- to eight-weekold female ND4 mice (Harlan) weighing 21-26 g. The density was established by counting cells in DB, LB and Wh preparations, and compartments in IWr preparations. Preparations were also plated at the time of inoculation to verify c.f.u. and phenotype. After 7 days, mice were euthanized, and spleen, liver and kidney, and in the timecourse study also brain, were removed, weighed and ground in $2 \mathrm{ml}$ sterile PBS in a sterile mortar and pestle. Aliquots of each organ were immediately plated on YPD agar containing $1 \mathrm{mM} \mathrm{CuSO}_{4}$ and incubated at $25{ }^{\circ} \mathrm{C}$ for 5 days. For time-course experiments, 24 mice were injected with DB cells and 24 with Wh cells. Then at various time points, three animals from each group were euthanized and analysed.

Construction of a strain harbouring GFP-SAT ${ }^{\mathbf{r}}$. An $M T L$-specific $450 \mathrm{bp}$ target sequence was generated by fusing two PCR fragments containing an internal XhoI site. The fragments were amplified with the pair of $5^{\prime}$ PCR primers a2f1 (5'-GCAAGAGATTAATAACCAAC$\left.3^{\prime}\right)$ and a2r1 (5'-GCAGATGGTCTTTGGTAACC-3'), and a pair of $3^{\prime}$ PCR primers a2f2 (5'-TGCCGCTTCCTATCGAGGC-3') and a2r2 $\left(5^{\prime}\right.$-GTCGAGGCTGTTTTGAGCC-3'). The PCR products were ligated with the pGEM-T Easy plasmid (Promega) to generate pI76.1, which was used as a basic backbone to generate the final GFP$\mathrm{SAT}^{\mathrm{r}}$ expression module. The GFP-TDH3 promoter fusion was generated as follows. Two fragments encompassing 700 bp downstream and $300 \mathrm{bp}$ upstream of the TDH3 coding region were amplified by PCR using Saccharomyces cerevisiae DNA as template. The primer pairs for the upstream region were TDF1 ( $5^{\prime}$ TAACTCGAGTCAGTTCGAGTTTATCATTATC-3') and TDR1 (5'TAATCTAGATTTGTTTGTTTATGTGTGTTTATTC-3'). Those for the downstream region were TDF2 (5'-TAATCTAGATAAGTGAATTTACTTTAATCTTGC-3') and TDR2 (5'-TAAAGATCTTCAGAATCGTTATCCTGGC- $3^{\prime}$ ). PCR products were digested with $\mathrm{Xba \textrm {I }}$ and ligated with the pGEM-T Easy plasmid (Promega) to generate PI78.1. The GFP ORF was amplified by PCR using pGFPURA3 as the template (a generous gift from Dr Judith Berman, University of Minnesota) (Gerami-Nejad et al., 2001) and the primers Gfcfxbf1 $\quad\left(5^{\prime}\right.$-TAACTAGAATGTCTAAAGGTGAAGAATTATTC- $\left.3^{\prime}\right)$ and Gfcfxbr1 (5'-TAATCTAGATTATTTGTACAATTCATCC-3'). The PCR product was digested with $\mathrm{XbaI}$ and subcloned into the $X b a$ I site of pI78.1 to derive pI82.10 for the TDH3-GFP fusion. The final plasmid (pI89.2) contained the GFP-SAT ${ }^{\mathrm{r}}$ module. The $1706 \mathrm{bp}$ GFP expression module was isolated by digesting pI82.10 with BglII and XhoI. The 1880 bp SAT ${ }^{\mathrm{r}}$ dominant marker was derived by PCR using pSFAF2 (a generous gift from Dr Joachim Morschhäuser, University of Würzburg) (Reuss et al., 2004) as a template and the primer pair CaSatF1 (5'-TTAAGATCTTCCATCATAAAATGTCGAGC-3') and CaSatR1 (5'-TTACTCGAGGAACTTCTTGCAGGACCACC- $3^{\prime}$ ). The PCR product was digested with BglII and XhoI. The GFP expression module and SAT ${ }^{\mathrm{r}}$ marker were simultaneously subcloned into the MTL genomic target plasmid pI78.1 to generate the plasmid pI89.2. A control plasmid pI188.1 was constructed in which a non-fluorescent version, CFP, was inserted in place of the functional GFP ORF. The former was constructed from an amplification product derived from pCFP-URA3 (a generous gift of Dr Judith Berman).

The clinical strain 35B11 was transformed with GFP-SAT ${ }^{\mathrm{r}}$ or CFPSAT $^{r}$. Approximately $5 \mu \mathrm{g}$ of pI89.2 or pI88.1 harbouring CFP-SAT ${ }^{\mathrm{r}}$ or GFP-SAT ${ }^{\mathrm{r}}$, respectively, was isolated by digestion with ApaI and SacI, and used for MTLa2-targeted integrative transformation. Transformants were selected on YPD agar plates supplemented with $25 \mu \mathrm{g}$ clonNAT ml ${ }^{-1}$ according to methods previously described (Srikantha et al., 2005). Transformants were verified by PCR and Southern blot hybridization (data not shown). Several independently derived GFP transformants were tested for GFP expression by fluorescence microscopy. Several independently derived control transformants containing CFP were tested for the absence of fluorescence. The fluorescent GFP-expressing strain Ca2-Gf2 and the non-fluorescent CFP-expressing strain Ca2-CF1 were selected for experimental use. Both strains were tested and found to undergo core switching normally (Lachke et al., 2000, 2002).

Cell viability. Cells were sonicated twice for $10 \mathrm{~s}$ on ice. Cells were pelleted, suspected in Hank's Buffered Saline (Gibco) and stained with $50 \mathrm{nM}$ SYTOX Orange (Invitrogen). Cells were imaged using a Bio-Rad MP2100 laser scanning confocal microscope connected to a Nikon TE2000 microscope. A $10 \mu \mathrm{m} Z$ series was collected at $0.25 \mu \mathrm{m}$ intervals and projected. Fluorescent and differential interference contrast images were merged for dead cell counting.

\section{RESULTS}

\section{Characterization of the cell preparations used in animal model experiments}

Our objective was to compare virulence among the switch phenotypes of $C$. glabrata using the mouse model of systemic infection (MacCallum \& Odds, 2005; Joly \& Yeni, 1999; de las Penas et al., 2003). To obtain cell preparations of each switch phenotype, stored DB cells of strain 35B11, a clinical isolate that was mating type a, were spread on agar plates containing YPD nutrient medium supplemented with $1 \mathrm{mM} \mathrm{CuSO}_{4}$ (Lachke et al., 2000). From these DB cultures, cells from homogeneous Wh, LB or IWr sectors or colonies (Fig. 1a) were plated on fresh agar and grown for 4 days, and several colonies homogeneous for each of 
(a)

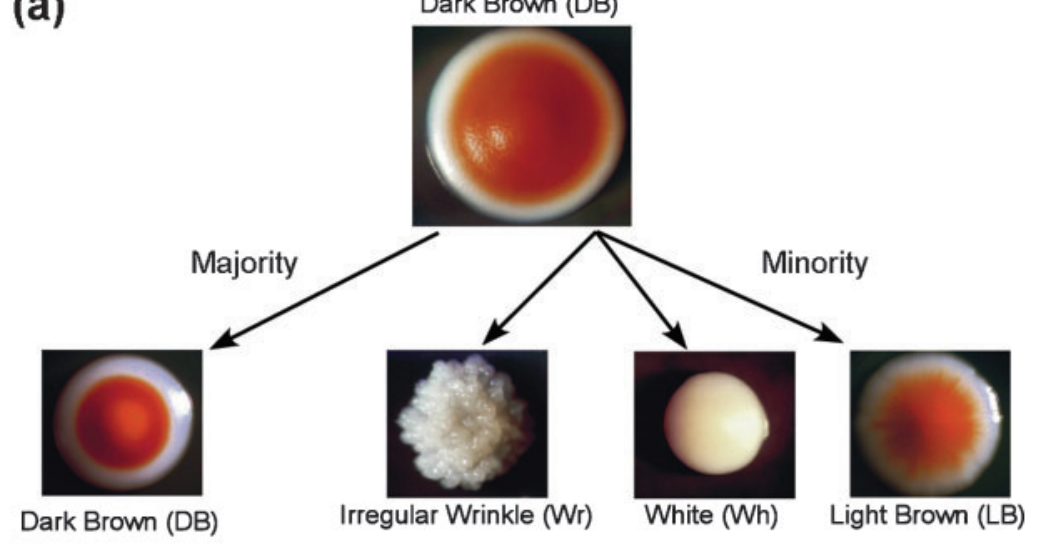

(b) Dark Brown (DB) phenotype development

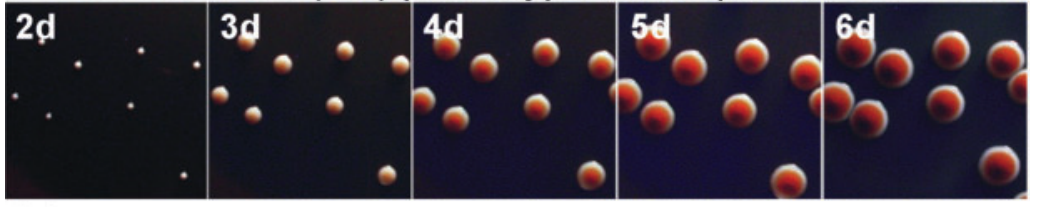

(c) Light Brown (LB) phenotype development

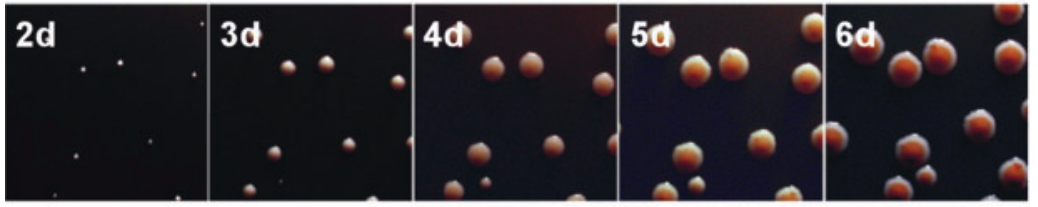

(d) White (Wh) phenotype development

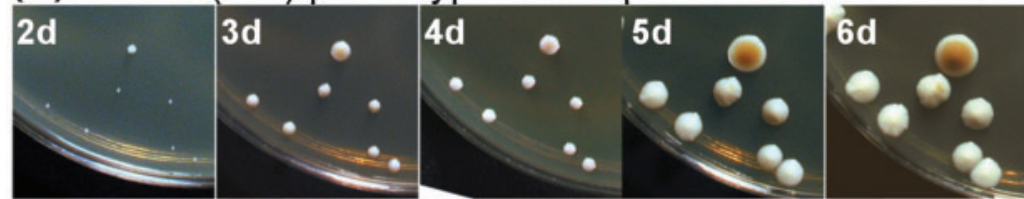

(e) Irregular wrinkle (IWr) phenotype development

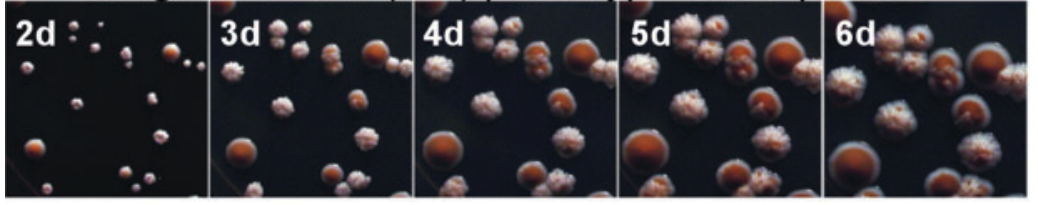

Fig. 1. The switch phenotype preparations used in the mouse model experiments. (a) Cells from a DB colony stored at $-80{ }^{\circ} \mathrm{C}$ were plated, and DB, LB, Wh and IWr colonies picked. (b, c, d, e) Cells from three colonies of each phenotype were in turn pooled and plated, and development of colonies was monitored over a 6-day period. Colonies grown for 5 days were pooled for injection into mice. The percentage of DB colonies emanating from original $\mathrm{DB}$ colonies was approximately $98 \%$; the percentage of LB colonies from original LB colonies was approximately $94 \%$; the percentage of Wh colonies from original $\mathrm{Wh}$ colonies was approximately $80 \%$; and the percentage of IWr colonies from original IWr colonies was approximately $70 \%$. the switch phenotypes were pooled for injection into the tail vein of outbred ND4 mice.

When cells from homogeneous 5-day DB or LB colonies were pooled and replated, on average 98 and 94\%, respectively, exhibited the original $\mathrm{DB}$ or $\mathrm{LB}$ phenotype (Fig. 1b, c, respectively). When cells from homogeneous Wh colonies were pooled and replated, on average $80 \%$ exhibited the original Wh phenotype (Fig. 1d). When cells from IWr colonies originating from DB cultures were pooled and replated, only $70 \%$ expressed the original $\mathrm{IWr}$ phenotype; the remaining $30 \%$ expressed the DB phenotype from which they were derived (Fig. 1e). This high level of reversion of IWr has been reported previously (Lachke et al., 2002). The different degrees of contamination by other switch phenotypes reflect the different rates of switching, as previously demonstrated (Lachke et al., 2000, 2002).

The generation times of DB, LB and Wh cells were tested under standard laboratory conditions. On agar, colonies grown for 5 days at $25{ }^{\circ} \mathrm{C}$ contained approximately the same number of cells, and were visually of similar size, suggesting similar rates of cell multiplication. IWr colonies contained lower concentrations, primarily as a result of a significant proportion of pseudohyphal growth (Lachke et al., 2002; Csank \& Haynes, 2000). In liquid culture at $25{ }^{\circ} \mathrm{C}$, the generation times for $\mathrm{DB}, \mathrm{LB}$ and Wh cells were 1.7, 1.8 and $1.9 \mathrm{~h}$, respectively. At $37^{\circ} \mathrm{C}$, the generation times were 1.6, 1.7, $1.8 \mathrm{~h}$, respectively. These results were the same in two independent experiments. Because of 
clumping, the generation time for IWr was more difficult to assess, but estimates ranged between 1.8 and $2.0 \mathrm{~h}$.

\section{Colonization in the mouse model for systemic infection}

Five mice received injections of pooled cells of each switch phenotype. Since IWr colonies contained pseudohyphae that clumped (Lachke et al., 2002), cells from these colonies were sonicated, resulting in approximately $60 \%$ singlets, $20 \%$ doublets and $20 \%$ triplets. An analysis with SYTOX Orange, a dye that identifies dead cells (Truernit \& Haseloff, 2008), revealed that $2 \%(n=1325)$ of the cells were dead before sonication and $5 \%(n=1530)$ after sonication. Seven days after injection, the mice were euthanized, the spleen, liver and kidneys removed and weighed, and the c.f.u. per gram of organ measured. The selection of organs was based on the observations made by de las Penas et al. (2003) on the intensity of colonization after 7 days. A comparison of colonization among the switch phenotypes is presented by organ (Fig. 2).

Kidneys. The level of colonization of the three core phenotypes (Wh, LB and DB) was lower in the kidneys than in the liver and spleen (Fig. 2). The level of colonization of the kidneys by IWr was higher than that of the three core phenotypes. It was also slightly higher than IWr colonization of the liver, but more than threefold lower than that of the spleen (Fig. 2). The hierarchy of kidney colonization by the four tested switch phenotypes was $\mathrm{IWr}>\mathrm{DB}>\mathrm{LB}>\mathrm{Wh}$.

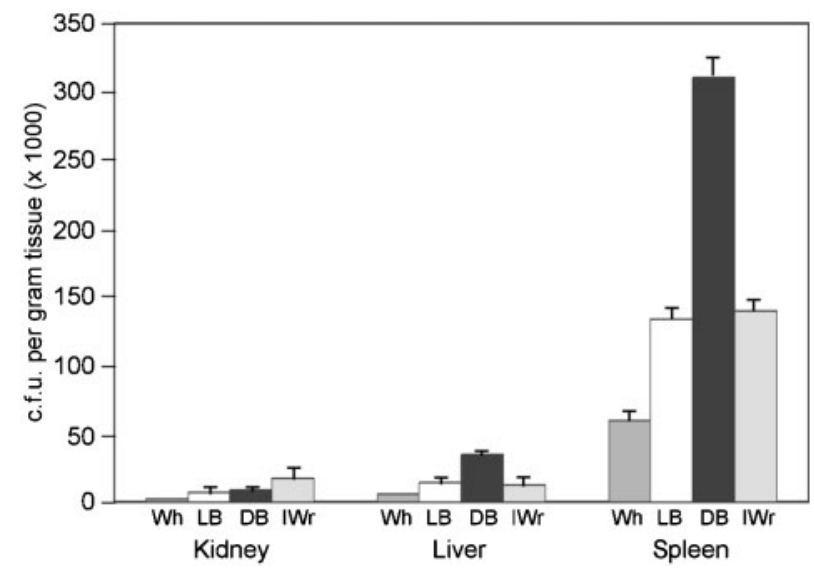

Fig. 2. The spleen is the most intensely colonized organ and DB the most intensely colonizing phenotype, in the mouse model of systemic infection. Mice received $2 \times 10^{7}$ cells of each phenotype, and were killed after 7 days, their organs weighed and the density of cells measured. Five mice were inoculated for each phenotype. Measurements were normalized to c.f.u. per gram of tissue. Means \pm SD (error bars) are shown.
Liver. The level of colonization of the three core phenotypes (Wh, LB and $\mathrm{DB}$ ) was higher in the liver than in the kidneys, but far lower than that in the spleen (Fig. 2). Colonization of the liver by DB was higher than that of all other tested phenotypes, 5.5 times that of Wh, 2.4 times that of LB and 2.7 times that of IWr (Fig. 2). The hierarchy of liver colonization was $\mathrm{DB}>\mathrm{LB} \approx \mathrm{IWr}>\mathrm{Wh}$.

Spleen. The level of colonization of the spleen was higher than that of the liver or kidney for all four tested phenotypes (Fig. 2). The hierarchy of spleen colonization was $\mathrm{DB}>\mathrm{IWr} \approx \mathrm{LB}>\mathrm{Wh}$. The level of colonization of $\mathrm{DB}$ in the spleen was approximately 2.5 times than that of IWr and LB, and six times higher than that of Wh. DB colonization of the spleen was eight times higher than $\mathrm{DB}$ colonization of the liver and 40 times higher than DB colonization of the kidney (Fig. 2).

The preceding colonization results were based on the number of c.f.u. per gram of organ tissue. Because the three major target organs, kidney, liver and spleen, differ dramatically in size, we employed an alternative measure of comparison, c.f.u. per organ. The mean weights of the two kidneys, the liver and the spleen were $321 \pm 44$, $2311 \pm 136$ and $95 \pm 18 \mathrm{mg}$, respectively. For DB colonization, the mean number of c.f.u. for the two kidneys was 2760, for the liver 80154 and for the spleen 29193. Hence, the liver, which has 24 times the mass of the spleen, was colonized in toto by 2.5 times as many C. glabrata cells. The hierarchy of DB colonization after 7 days based on c.f.u. per organ was liver>spleen>kidneys, whereas for $\mathrm{DB}$ colonization based on c.f.u. per milligram of tissue it was spleen $>$ liver $>$ kidneys.

\section{Maintenance of injected phenotypes}

The above experiments demonstrated that DB cells, when injected into the tail vein of a mouse, caused far higher levels of colonization in the two major target organs, liver and spleen, than the other switch phenotypes. The experimental protocol however, did not test whether injected DB cells retained that phenotype upon colonization, or whether the other injected switch phenotypes retained theirs. We therefore measured the proportion of colonizing cells that expressed the original colony phenotype in the kidney, liver and spleen. No significant changes were observed for injected DB, LB or Wh cells after 7 days in the host (Table 1). In the case of IWr, there was no significant change in the kidneys, but there was a significant decrease in the proportion of IWr cells in the liver and spleen (Table 1) (non-parametric Mann-Whitney $\mathrm{U}$ test, $P=0.0121$ in both cases). This was accompanied by a compensating increase in the proportion of $\mathrm{DB}$ cells, the core phenotype from which the IWr cells originated (Table 1). The latter results do not, however, discriminate between selective colonization by a minority DB cells that contaminate the original IWr preparation that was injected and an increase in the frequency of switching from IWr to DB. 
Table 1. Injected switch phenotypes remain relatively stable through 7 days of organ colonization.

\begin{tabular}{|lcccr|}
\hline \multirow{2}{*}{ Organ } & \multicolumn{4}{c|}{ Injected phenotype } \\
\cline { 2 - 5 } & DB $(\mathbf{9 8} \%)^{*}$ & LB $(\mathbf{9 4} \%)^{*}$ & Wh $(\mathbf{8 0} \%)^{*}$ & IWr $(\mathbf{7 0} \%)^{*}$ \\
\hline Kidney $\dagger$ & $98 \pm 2 \mathrm{DB}$ & $89 \pm 9 \mathrm{LB}$ & $78 \pm 7 \mathrm{Wh}$ & $66 \pm 6 \mathrm{IWr}$ \\
& & & $(22 \pm 7 \mathrm{DB})$ & $(33 \pm 6 \mathrm{DB})$ \\
Liver $\dagger$ & $97 \pm 2 \mathrm{DB}$ & $94 \pm 2 \mathrm{LB}$ & $76 \pm 5 \mathrm{Wh}$ & $43 \pm 3 \mathrm{IWr}$ \\
& & & $(21 \pm 5 \mathrm{DB})$ & $(54 \pm 3 \mathrm{DB})$ \\
Spleen $\dagger$ & $98 \pm 2 \mathrm{DB}$ & $90 \pm 3 \mathrm{LB}$ & $72 \pm 5 \mathrm{Wh}$ & $42 \pm 6 \mathrm{IWr}$ \\
& & & $(23 \pm 4 \mathrm{DB})$ & $(54 \pm 6 \mathrm{DB})$ \\
& & & & \\
\hline
\end{tabular}

${ }^{*}$ Percentage of the major phenotype in the inoculum, assessed by plating experiments.

$\dagger$ The proportion of the cells colonizing each organ that expressed the original injected phenotype, assessed by plating experiments. Means \pm SD are shown for five mice for each phenotype.

\section{The colonization advantage of DB is independent of initial copper content}

The preceding mouse model experiments were performed with cell preparations of the different switch phenotypes obtained from colonies of strain 35B11 grown on agar containing $1 \mathrm{mM} \mathrm{CuSO}_{4}$ in order to identify colonies with homogeneous phenotypes (Fig. 1). Since DB cells of strain $35 \mathrm{~B} 11$ grown in agar containing $1 \mathrm{mM} \mathrm{CuSO}_{4}$ contain over 10 times the amount of copper measured in Wh cells grown under similar conditions (T. Srikantha, K. J. Daniels \& D. R. Soll, unpublished data), comparisons were made of the colonization of $\mathrm{DB}$ and Wh cells grown in the absence of $\mathrm{CuSO}_{4}$ and in $1 \mathrm{mM} \mathrm{CuSO}_{4}$. Five mice were examined for each of the tested conditions. $\mathrm{DB}$ cells grown in the presence or absence of $1 \mathrm{mM} \mathrm{CuSO}_{4}$ colonized kidney, liver and spleen to approximately the same extent (Fig. 3). Similar results were obtained for Wh cells (Fig. 3). These results demonstrate that the advantage that $\mathrm{DB}$ cells have in colonization is independent of copper content.

\section{DB cells retain a colonization advantage in mixed infections}

To test whether DB exerted its advantage in co-infections, a $50: 50$ mixture of $\mathrm{DB}$ and Wh cells of strain $35 \mathrm{~B} 11$ was injected into mice, and the phenotypes of infecting cells were assessed in liver, kidney and spleen after 7 days. Phenotypes were assessed by analysing the colonies formed from yeast cells in organ macerates. The major phenotype $(75-83 \%)$ in the kidney, liver and spleen was DB (Fig. 4a). Plating experiments with the initial 50:50 mixture verified that the inoculum was made up of $50 \%$ Wh and $50 \% \mathrm{DB}$ cells. Similar results were obtained when a 50:50 mixture of DB and IWr cells was injected; the major phenotype (DB) varied between 82 and $87 \%$ in the three target organs (Fig. 4b). In this case, because the initial IWr cell preparation was sonicated, IWr cells were tested for

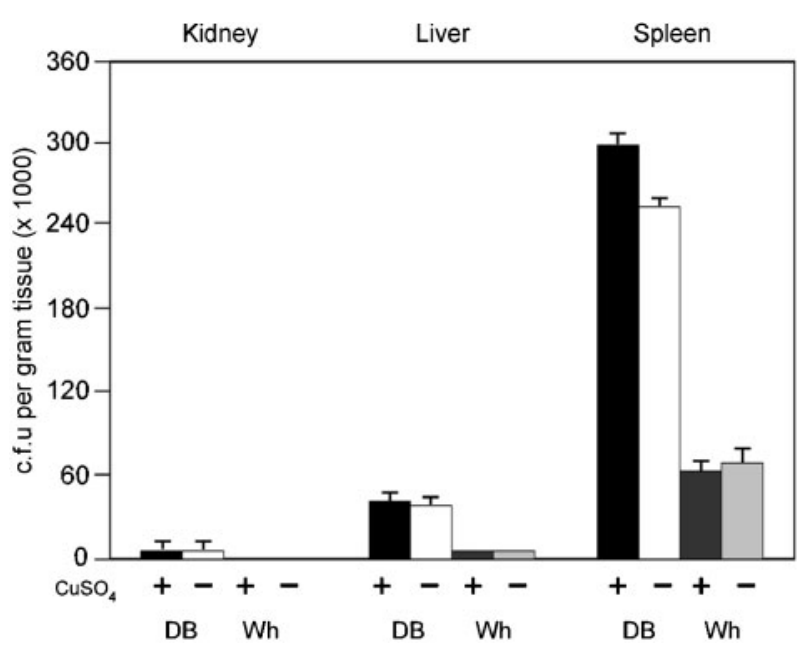

Fig. 3. Growth on agar supplemented with $1 \mathrm{mM} \mathrm{CuSO}_{4}$ is not necessary for the DB advantage in organ colonization. A comparison was performed between cells grown on nutrient agar containing $1 \mathrm{mM} \mathrm{CuSO}_{4}(+)$ and cells grown on unsupplemented nutrient agar $(-)$ for organ colonization. The density of colonization was normalized to that of maximum colonization (i.e. in the spleen of hosts injected with DB grown on agar supplemented with $1 \mathrm{mM}$ $\mathrm{CuSO}_{4}$ ). Three mice were injected for each condition. Means $\pm \mathrm{SD}$ (error bars) are shown.

viability with SYTOX Orange. Prior to sonication, $2 \%$ of cell compartments were dead; after sonication, $5 \%$ were dead, a difference that did not affect the outcome.

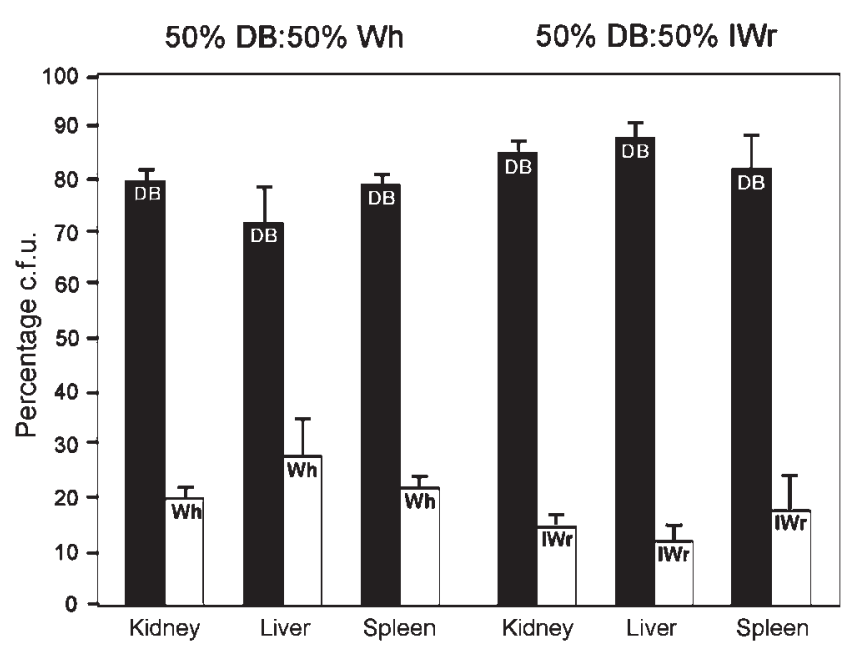

Fig. 4. In mixed-phenotype infections, DB retains its advantage over Wh or IWr in organ colonization. To test for competitiveness, DB cells were mixed 50:50 with Wh or IWr and injected into mice. Liver, kidney and spleen were then analysed for the proportions of switch phenotypes colonizing them. Five mice were injected with each combination. Means \pm SD (error bars) are shown. 
To demonstrate that these results were not due to increased rates of switching from Wh to DB or IWr to DB (Lachke et al., 2002), strain 35B11 was transformed with a construct containing the GFP ORF (GFP) under the control of the constitutive S. cerevisiae TDH3 promoter (http://db.yeastgenome.org/cgi-bin/locus.pl?locus $=$ tdh3; Delgado et al., 2001) (Fig. 5a). This GFP expression cassette was targeted to the MTL1 locus, to generate strain Ca2-GF2. Strain 35B11 was similarly transformed with a mutant GFP, generating the nonfluorescent control strain $\mathrm{Ca} 2-\mathrm{CF} 1$. In vitro, $\mathrm{Wh}$ and $\mathrm{DB}$ cells of the GFP-expressing strain Ca2-GF2 were constitutively fluorescent, whereas $\mathrm{Wh}$ and $\mathrm{DB}$ cells of the control strain Ca2-CF1 did not produce a fluorescent signal (Fig. 5b). When fluorescent Wh cells of strain Ca2GF2 were mixed 50:50 with non-fluorescent DB cells of strain $\mathrm{Ca} 2-\mathrm{CF} 1$, the majority of cells that colonized the kidney, liver and spleen of two test mice (Exp. 1 and Exp. 2) were non-fluorescent (Fig. 5c). When fluorescent DB cells of strain Ca2-GF2 were mixed 50:50 with nonfluorescent Wh cells of strain Ca2-CF1, the majority of cells that colonized the kidney, liver and spleen of two test mice were fluorescent (Fig. 5d). These results demonstrate that when DB cells are co-injected with Wh cells, the majority of cells that colonize the kidney, liver and spleen represent the original DB population.

(a) GFP expression cassette

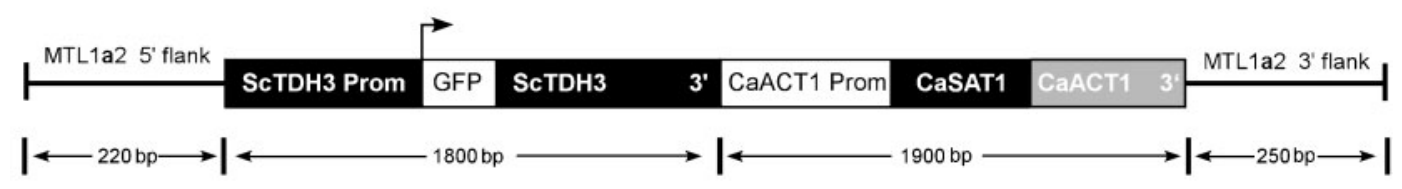

(b) Fluorescence in GFP-expressing cells
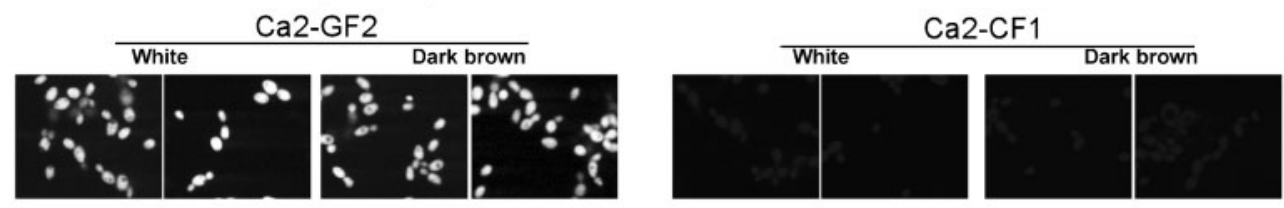

(c) Fluorescent Wh $\square$ vs. Non-fluorescent DB

(d) Non-fluorescent Wh $\square$ vs. Fluorescent DB
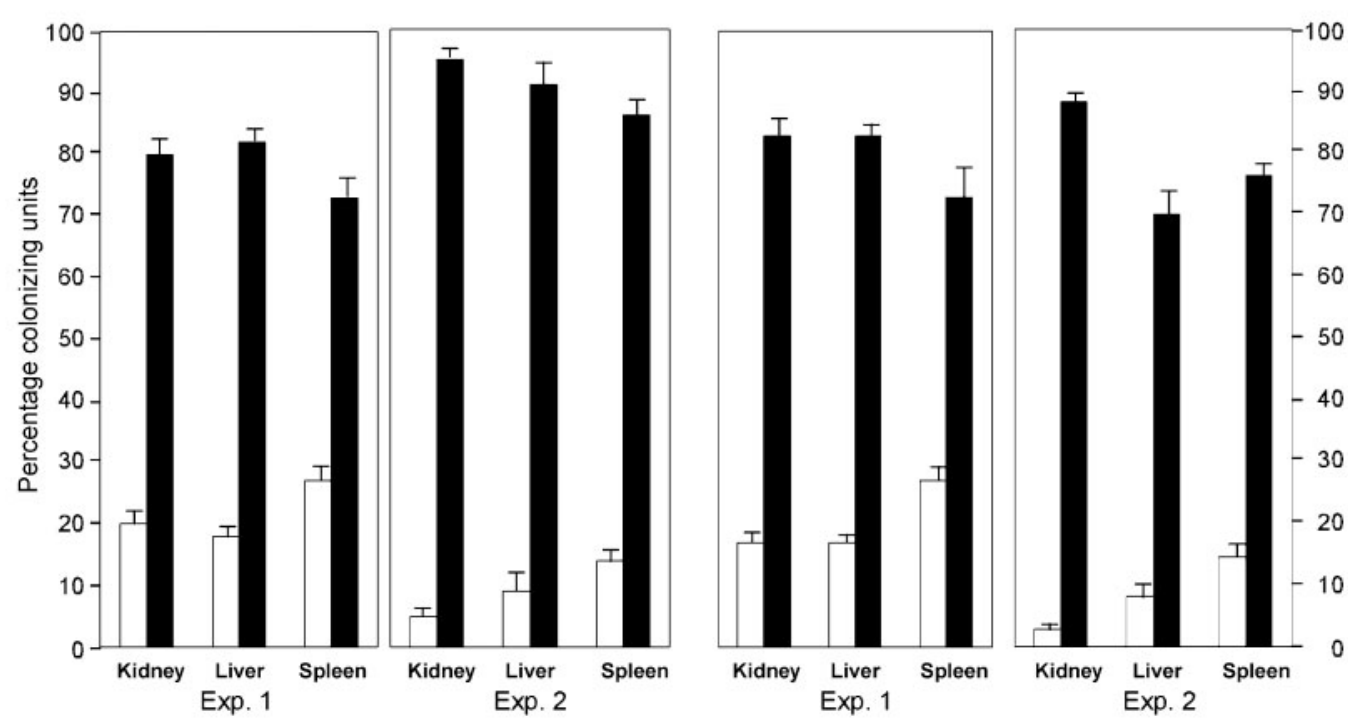

Fig. 5. The use of a strain constitutively expressing GFP confirms that DB has an advantage over Wh in $50: 50$ co-injection experiments. (a) GFP expression cassette used to generate strain Ca2-GF2, a derivative of strain 35B11. (b) Demonstration that cells of strain Ca2-GF2 are constitutively fluorescent in the Wh and DB phenotype, whereas cells of strain Ca2-CF1, transformed with a functionless GFP, are non-fluorescent. (c) The results of a 50:50 co-injection experiment with fluorescent Wh Ca2-GF2 cells and non-fluorescent DB Ca2-CF1 cells. (d) The results of a 50:50 co-injection experiment with nonfluorescent Wh Ca2-CF1 and fluorescent DB Ca2-GF2 cells. The results of two separate experiments are presented for each of the combinations in (c) and (d). In each experiment, three mice were injected with each combination. Means $\pm S D$ (error bars) are shown. 


\section{Time-course of organ colonization and clearing}

The preceding mouse model experiments, which were performed according to the methods of de las Penas et al. (2003), involved analyses of organ colonization at a single defined time point of 7 days following tail injection. Since the injection protocol does not result in host death, one might expect organ clearing and hence changes in organ colonization over time. We therefore performed an experiment in which organ colonization was analysed as a function of time for injected Wh cells or injected DB cells. The body locations analysed were blood, spleen, liver, kidney and brain. Because both DB and Wh had cleared from the blood by the first time point $(2 \mathrm{~h})$, the data for blood are not shown.

Both DB and Wh cells reached maximum or nearmaximum concentrations in the kidney, liver and spleen within $2-4$ h post-injection (Fig. 6a, d, g, respectively). The maximum concentrations attained by $\mathrm{DB}$ and Wh cells (Fig. 6a, d, g, respectively) followed the same hierarchy observed in the single time point experiments: spleen $>$ liver $>$ kidney (Fig. 7a, b). Initial colonization in the kidney was similar for $\mathrm{DB}$ and Wh (Fig. 6a), but initial DB colonization was much higher than initial Wh colonization in the two major target organs liver and spleen (Fig. 6d, g), as was observed in the single time point (7-day) experiments (Fig. 2). Clearing of DB and Wh cells occurred in all three major target organs (Fig. 6a, d, g; Fig. 7a, b). At 7 days, the decrease in DB colonization of the kidney, liver and spleen was 24-, 52- and 16-fold, respectively, and the decrease in Wh colonization 90-, 320- and 9-fold, respectively (Fig. 7a, b). Clearing of Wh from liver and spleen was therefore much faster than clearing of DB. During clearing in kidney, liver and spleen, Wh cells dominated at all time points in animals injected with a Wh cell preparation (Fig. 6b, e, h, respectively), and DB cells dominated at all time points in animals that received a DB cell preparation (Fig. 6c, f, i, respectively). After 1 day there was slight enrichment of DB in the organs of animals that received Wh cells (Fig. 6 b, e, h).

The time-course of brain colonization differed dramatically from that of the other three organs. Colonization of DB cells was low for at least the first $6 \mathrm{~h}$, then increased gradually to a maximum after 5-7 days (Fig. 7a). Colonization then decreased so that by 14 days, DB cells had decreased by more than 34-fold (Fig. 7a). Unlike DB cells (Fig. 7a), injected Wh cells did not show a transient increase in brain colonization between 1 and 7 days (Fig. $7 b)$. During both the slow colonization of the brain (05 days) and subsequent clearing (5-14 days), the majority of colonizing cells exhibited the DB phenotype of the original cell preparation (Fig. 6b).

\section{DISCUSSION}

Most strains of C. glabrata switch spontaneously and reversibly among four major core phenotypes, and between each core phenotype and the IWr phenotype (Lachke et al., 2000, 2002; Brockert et al., 2003). Yet the core phenotype $\mathrm{DB}$ is expressed by a majority of clinical isolates. This suggested to us that expressing DB provides cells with an advantage in colonizing the human host. If true, then one or more phenotypic characteristics associated with the DB phenotype must provide this advantage, and identification of these characteristics could provide insights into $C$. glabrata virulence. For that reason, we tested whether cells expressing the DB phenotype had an advantage over other switch phenotypes in colonizing the organs of mice in the model of systemic infection (de las Penas et al., 2003). The results presented here demonstrate that this is indeed the case, providing us with an assay, organ colonization, to identify in future mutational analyses the phenotypic characteristic(s) of DB that provides this advantage.

Our initial results, employing the procedures of de las Penas et al. (2003), revealed that the spleen, liver and kidney were major targets of colonization 7 days postinjection for the four tested phenotypes DB, LB, Wh and IWr, and, as de las Penas et al. (2003) demonstrated, the intensity of colonization, measured as c.f.u. per gram of tissue, was highest in the spleen, then the liver and finally the kidney. Our results, and these of de las Penas et al. (2003), are not consistent with the study by Brieland et al. (2001), but that may be explained by the fivefold higher inoculum of yeast of Brieland et al. and a very different mouse host.

On the basis of c.f.u. per organ, colonization of the liver was the highest, the spleen the second highest and the kidneys the lowest. One might assume, however, that colonization measured as c.f.u. per gram of tissue is more indicative of the damage that might be caused by the colonizing yeast. More importantly, and the intent of this study, we found that in the two major target organs, spleen and liver, colonization by $\mathrm{DB}$ was far higher than that of the three other tested switch phenotypes after 7 days. DB colonization of the spleen was more than two times that of LB and IWr, and over five times that of Wh. Furthermore, competition experiments demonstrated unequivocally that if equal numbers of DB and Wh, or DB and IWr cells, were co-injected into the mouse, the original DB cells predominated in the three target organs. At least in vitro, no significant differences were observed in the generation time of the core phenotypes, suggesting that it was differential retention, not differential growth rates, that were the basis of our results.

Although the initial results of our animal model experiments supported the hypothesis that the switch phenotype DB is most commonly expressed in human hosts because of a colonization advantage, we became concerned that this result was limited to a single sampling time point, and might not be representative, given that because C. glabrata does not kill its host in the mouse model of systemic infection employed here, the cells in infected organs may have complex clearing dynamics. We therefore performed a 


\section{Comparative Colonization}
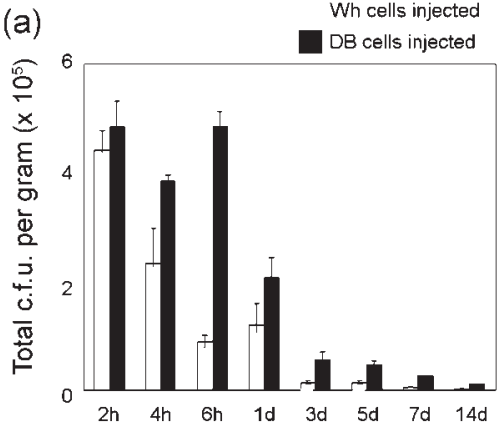

(d)

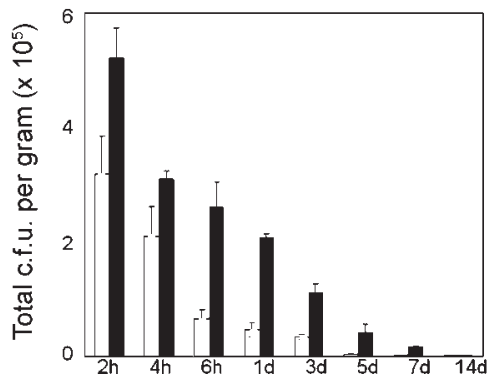

(g)

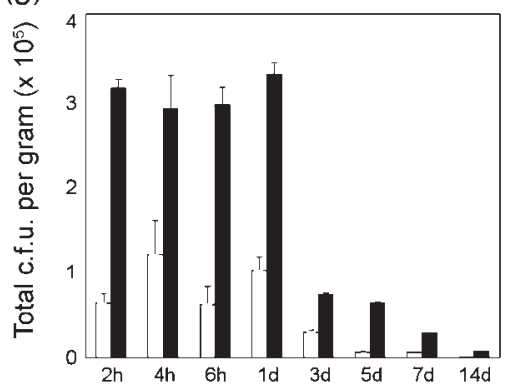

(j)

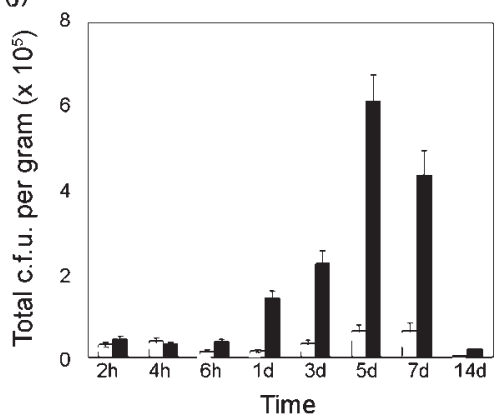

Injected Cells: Wh
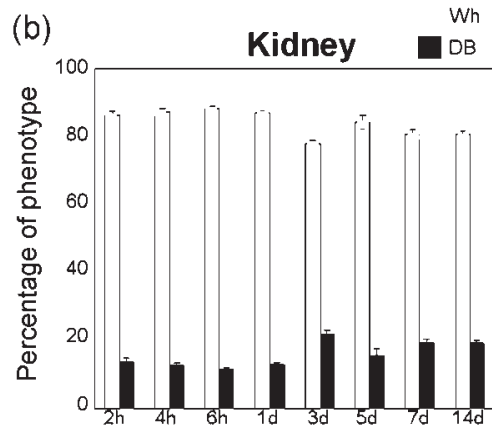

(e)

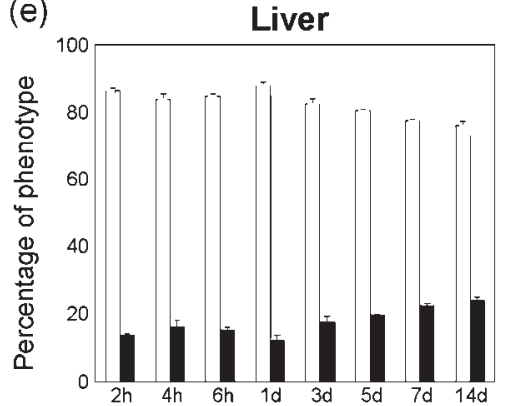

(h)

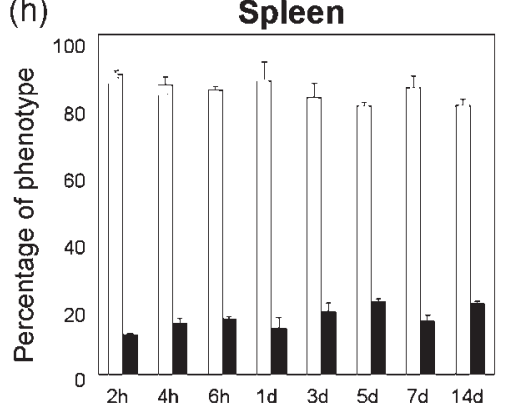

(k)

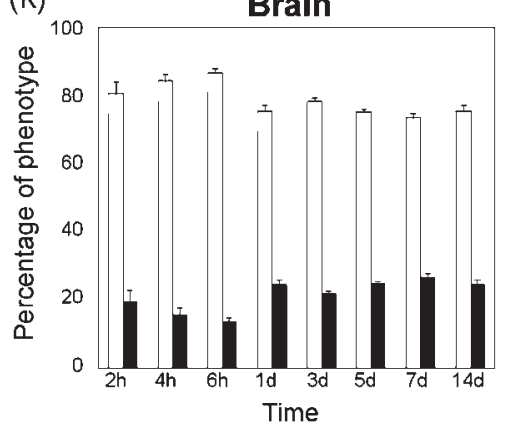

Injected Cells: DB

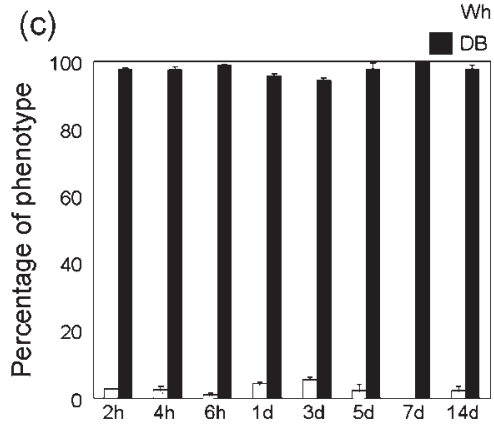

(f)

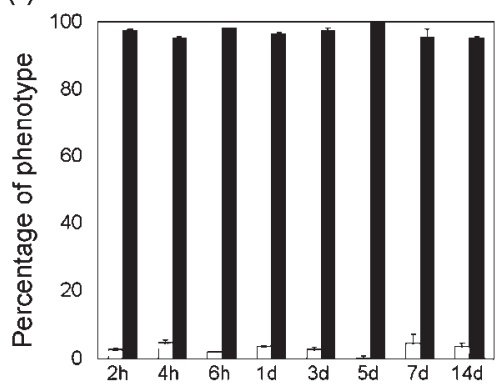

(i)

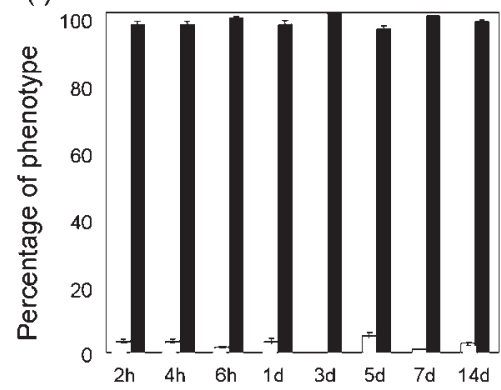

(I)

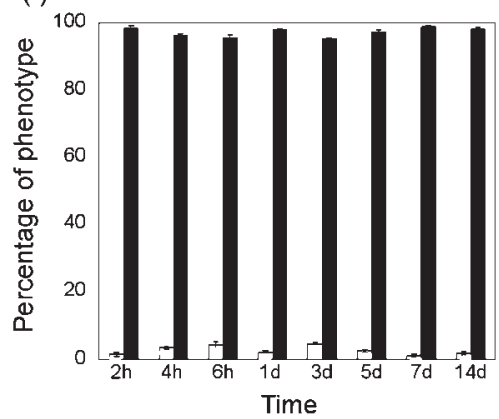

Fig. 6. Time-course of organ colonization of $D B$ and Wh cells in the mouse model of systemic infection. Sets of mice were injected with either Wh or DB cells. At the indicated time points, three mice from each set were euthanized and organs were analysed for colonization. For each organ, the mean density of colonization by Wh and DB is compared (first column), the proportion of colonizing Wh and DB cells is compared for mice injected with Wh cells (second column), and the proportion of DB and Wh cells is compared for mice injected with DB cells. In each experiment, 24 mice were injected with DB and 24 with Wh cells. Means $\pm \mathrm{SD}$ (error bars) are shown.

time comparison of DB and Wh colonization, and found differences in initial colonization versus 7-day colonization for the kidney, liver and spleen. Colonization by DB of the kidney was similar to that of Wh after $2 \mathrm{~h}$; colonization of the liver by DB was over $50 \%$ higher than that by Wh after $2 \mathrm{~h}$; and colonization of the spleen by DB was over five 
(a) $\mathrm{DB}$

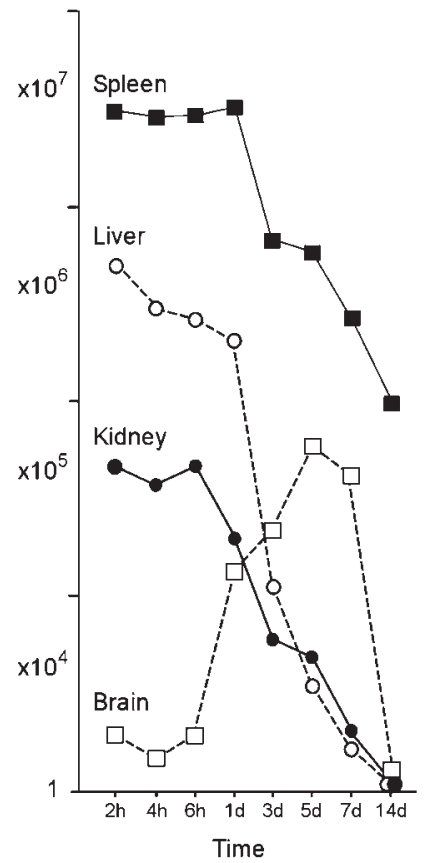

(b) White

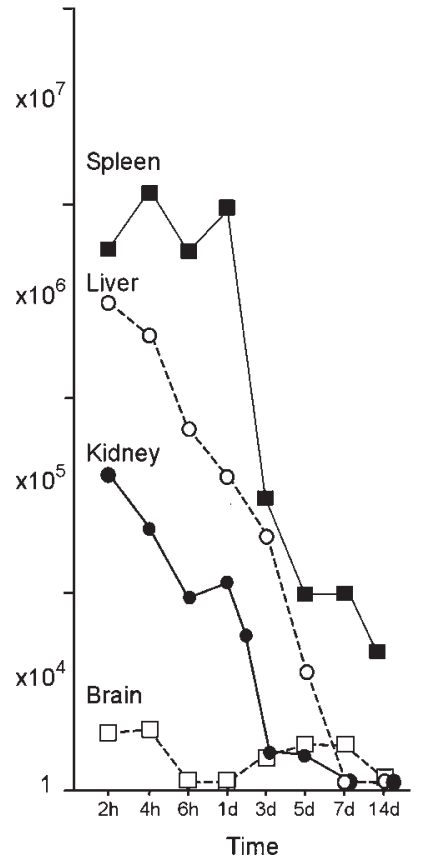

Fig. 7. Graphic representation of colonization and clearing of DB and Wh reveals the $\mathrm{DB}$ advantage and the selective colonization of the brain by $\mathrm{DB}$, but not Wh, between 1 and 7 days post injection. In each experiment, 24 mice were injected with DB and 24 with Wh cells. The mean numbers of mice at each time point are plotted.

times higher than that by Wh after $2 \mathrm{~h}$. After 5 days, when colonization by DB and Wh cells had decreased dramatically, in the kidney, liver and spleen, DB colonization was three, seven and seven times higher than Wh colonization, respectively. For the kidney and liver, the results suggested that Wh cells were cleared from these organs faster than DB cells. However, during clearing of Wh cells, there was only a small increase in the proportion of DB cells, suggesting that selectivity occurs both early in colonization and during clearing. Most importantly, however, the time comparison data supported the general conclusion based on the single time point experiments at 7 days (Figs 2, 3, 4) that DB maintains a colonization advantage over other switch phenotypes, in this case during the clearing process. The data also demonstrate that the hierarchy of organ colonization is spleen $>$ liver $>$ kidney for both $\mathrm{DB}$ and Wh cells as early as $2 \mathrm{~h}$ after injection, and that that hierarchy remains after 7 days.

The speed of organ colonization by DB cells supports the suggestion that DB dominance cannot be due to enrichment through switching and/or differential growth rates of switch phenotypes. Rather, the advantage must be due to an expressed cell characteristic, for instance selective adhesion to target organs or greater resistance to white cell phagocytosis or killing. The advantage of DB cells in either case could be the result of differences in cell surface proteins or other stress-resistance proteins (Weig et al., 2001; Haynes, 2001; Srikantha et al., 2005; Filler, 2006). C. glabrata expresses adhesin genes, most notably those in a family of epithelial adhesins, the EPAs (Cormack et al., 1999). Castano et al. (2005) have shown that certain genes in this family are regulated by telomeric silencing and that mutations in two genes involved in telomeric silencing, SIR1 and RIF1, result in increases in adherence to epithelial cells and increased kidney colonization. Simultaneous deletion of HYR1, a cell wall protein, and EPA1, EPA2 and EPA3, results in decreases in kidney colonization, but not liver or spleen colonization (de las Penas et al., 2003). The possibility must therefore be entertained that modulation of the expression of EPA genes by switching, possibly through metastable changes in telomere length, may be involved in the differences in organ colonization observed between switch phenotypes. This possibility is now being investigated.

We found that the spleen represented the most heavily colonized organ in the mouse model, and that DB was the most intensely colonizing switch phenotype. Colonization by DB cells was restricted to the red pulp of the spleen (K. J. Daniels, T. Srikantha \& D. R. Soll, unpublished observations). The spleen functions as a blood-filtering system, removing senescent red blood cells and microbes, the latter phagocytosed by macrophages inhabiting a marginal zone between the white and red pulp (Kraal, 1992). Since the spleen drains into the liver through the hepatic portal vein, we considered a scenario in which $C$. glabrata cells first accumulated in the spleen, then moved to the liver. However, because maximum colonization was attained simultaneously in both organs by $2 \mathrm{~h}$, then appeared to clear faster in the liver than the spleen, this scenario does not seem plausible. It is possible that the architecture of blood flow and the filtration process in the spleen are the reasons that this organ is immediately the most densely colonized for all tested switch phenotypes. But if that were simply the case, one might expect the spleen to be the major target organ for C. albicans colonization as well. Earlier experiments, however, have not demonstrated this to be the case (MacCallum \& Odds, 2005).

In contrast to the colonization dynamics of $\mathrm{DB}$ and $\mathrm{Wh}$ cells in the spleen, liver and kidneys of C. glabrata, colonization of the brain was very low for the first $6 \mathrm{~h}$ following injection, then gradually increased, reaching a maximum at 5-7 days. Colonization then decreased. Brieland et al. (2001) also found a transient increase in brain colonization, which, however, peaked 3 days postinjection. C. albicans colonization of the brain also begins to increase several days after injection, again differing markedly from the immediate colonization of the major target organs (MacCallum \& Odds, 2005). Even though the dynamics of brain colonization by C. glabrata differed from those of other target organs, DB still exhibited a major colonization advantage over Wh. In fact, there was no transient increase by Wh cells in brain colonization 
between one and 14 days, as there was by DB cells. These results indicate that whether colonization maxima are reached in hours or in days, DB exhibits a colonization advantage over other switch phenotypes.

In summary, our results demonstrate that $\mathrm{DB}$, the switch phenotype most commonly expressed by clinical isolates, has a distinct advantage over other core switching phenotypes and the IWr switch phenotype in colonizing the target organs in the mouse model of systemic infection. We have shown that this advantage is not related to either brown coloration or copper accumulation. It is manifested within hours of injection into the host, which indicates that it is not due to enrichment or differential growth, but rather to a characteristic, likely of the cell exterior, that provides a greater homing ability, perhaps through selective adhesion. The advantage is also revealed in clearing, and hence may also be related to white blood cell interactions. The advantage is also manifested in the far slower colonization of the brain. Elucidating the molecular basis of the DB advantage over other switch phenotypes may provide us with insights into the major virulence factors associated with organ colonization. Our results underscore the need in future studies to consider the effects of switching on the biology and virulence of C. glabrata, and to establish the switch phenotype of the strain being used by researchers in a particular study.

\section{ACKNOWLEDGEMENTS}

This research was supported by National Institutes of Health grant DE014219. The authors also wish to thank Terra Simon for her help with different aspects of this project.

\section{REFERENCES}

Brieland, J., Essig, D., Jackson, C., Frank, D., Lebenberg, D., Menzel, F., Arnold, B., DiDomenico, B. \& Hare, R. (2001). Comparison of pathogenesis and host immune responses to Candida glabrata and Candida albicans in systemically infected immunocompetent mice. Infect Immun 69, 5046-5055.

Brockert, P. J., Lachke, S. A., Srikantha, T., Pujol, C., Galask, R. \& Soll, D. R. (2003). Phenotypic switching and mating type switching of Candida glabrata at sites of colonization. Infect Immun 71, 71097118.

Castano, I., Pan, S.-J., Zupancic, M., Henniquin, C., Dujon, B. \& Cormack, B. P. (2005). Telomere length control and transcriptional regulation of subtelomeric adhesins in Candida albicans. Mol Microbiol 55, 1246-1258.

Cormack, B. P., Ghori, N. \& Falkow, S. (1999). An adhesion of the yeast pathogen Candida glabrata mediating adherence to human epithelial cells. Science 285, 578-582.

Csank, C. \& Haynes, K. (2000). Candida glabrata displays pseudohyphal growth. FEMS Microbiol Lett 189, 115-120.

de las Penas, A., Pan, S.-J., Castano, I., Alder, J., Cregg, R. \& Cormack, B. P. (2003). Virulence-related surface glycoproteins in the yeast pathogen Candida glabrata are encoded in subtelomeric clusters and subject to RAP1- and SIR-dependent transcriptional silencing. Genes Dev 17, 2245-2258.

Delgado, M. L., O’Connor, J. E., Azorin, I., Renau-Piqueras, J., Gil, M. L. \& Gozalbo, D. (2001). The glyceraldehyde-3-phosphate dehydrogenase polypeptides encoded by the Saccharomyces cerevisiae TDH1, TDH2 and TDH3 genes are also cell wall proteins. Microbiology 147, 411-417.

Filler, S. G. (2006). Candida-host cell receptor-ligand interactions. Curr Opin Microbiol 9, 333-339.

Gerami-Nejad, M., Berman, J. \& Gale, C. A. (2001). Cassettes for PCR-mediated construction of green, yellow, and cyan fluorescent protein fusions in Candida albicans. Yeast 18, 859-864.

Haynes, K. (2001). Virulence in Candida species. Trends Microbiol 9, 591-596.

Joly, V. \& Yeni, P. (1999). Rodent models of Candida species. In Handbook of Animal Models of Infection, pp. 649-655. Edited by O. Zak \& M. A. Sande. New York: Academic Press.

Kraal, G. (1992). Cells in the marginal zone of the spleen. Int Rev Cytol 132, 31-74.

Kvaal, C. A., Srikantha, T. \& Soll, D. R. (1997). Misexpression of the white-phase-specific gene WH11 in the opaque phase of Candida albicans affects switching and virulence. Infect Immun 65, 4468-4475.

Kvaal, C., Lachke, S. A., Srikantha, T., Daniels, K. J., McCoy, J. \& Soll, D. R. (1999). Misexpression of the opaque phase-specific gene PEP1 (SAP1) in the white phase of Candida albicans confers increased virulence in a mouse model of cutaneous infection. Infect Immun 67, 6652-6662.

Lachke, S. A., Srikantha, T., Tsai, L., Daniels, K. J. \& Soll, D. R. (2000). Phenotypic switching in Candida glabrata involves phase-specific regulation of the metallothionein gene $M T-I I$ and the newly discovered hemolysin gene HLP. Infect Immun 68, 884-895.

Lachke, S. A., Joly, S., Daniels, K. J. \& Soll, D. R. (2002). Phenotypic switching and filamentation in Candida glabrata. Microbiology 148, 2661-2674.

Lockhart, S. R., Wu, W., Radke, J. B. \& Soll, D. R. (2005). Increased virulence and competitive advantage of $\mathbf{a} / \alpha$ over $\mathbf{a} / \mathbf{a}$ or $\alpha / \alpha$ offspring conserves the mating system of Candida albicans. Genetics 169, 1883-1890.

MacCallum, D. M. \& Odds, F. C. (2005). Temporal events in the intravenous challenge model for experimental Candida albicans infections in female mice. Mycoses 48, 151-161.

Reuss, O., Vik, A., Kolter, R. \& Morschhauser, J. (2004). The SAT1 flipper, an optimized tool for gene disruption in Candida albicans. Gene 341, 119-127.

Srikantha, T., Zhao, R., Daniels, K. J., Radke, J. \& Soll, D. R. (2005). Phenotypic switching in C. glabrata accompanied by changes in expression of genes with deduced functions in copper detoxification and stress. Eukaryot Cell 4, 1434-1445.

Truernit, E. \& Haseloff, J. (2008). A simple way to identify non-viable cells within living plant tissue using confocal microscopy. Plant Methods 4, 15.

Weig, M., Haynes, K., Rogers, T. R., Kurzai, O., Frosch, M. \& Mühlschlegel, F. A. (2001). A GAS-like gene family in the pathogenic fungus Candida glabrata. Microbiology 147, 2007-2019.

Edited by: K. Kuchler 